\title{
TARANIS-a Satellite Project Dedicated to the Physics of TLEs and TGFs
}

Cite as: AIP Conference Proceedings 1118, 3 (2009); https://doi.org/10.1063/1.3137711 Published Online: 05 May 2009

François Lefeuvre, Elisabeth Blanc, J. L. Pinçon, and TARANIS Team

\section{ARTICLES YOU MAY BE INTERESTED IN}

ASIM-an Instrument Suite for the International Space Station AIP Conference Proceedings 1118, 8 (2009); https://doi.org/10.1063/1.3137718

Coupling of Thunderstorms and Lightning Discharges to Near-Earth Space AIP Conference Proceedings 1118, 1 (2009); https://doi.org/10.1063/1.3137707

\section{Lock-in Amplifiers up to $600 \mathrm{MHz}$}
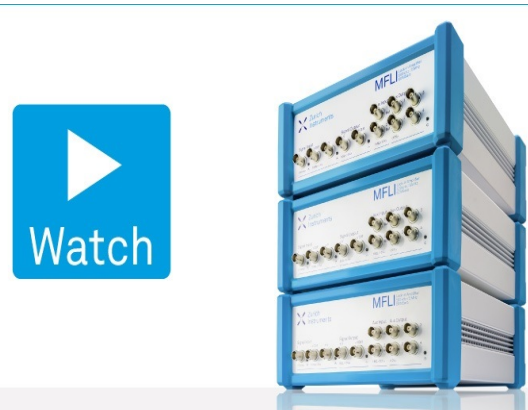


\title{
TARANIS - a Satellite Project Dedicated to the Physics of TLEs and TGFs
}

\author{
François Lefeuvre, Elisabeth Blanc ${ }^{\mathrm{b}}$, J.L. Pinçon ${ }^{\mathrm{a}}$, and TARANIS Team* \\ ${ }^{a}$ Laboratoire de Physique et Chimie de l'Environnement et de l'Espace-CNRS, Université d'Orléans, \\ 45071 Orléans cedex, France \\ ${ }^{b}$ Commissariat à l'Energie Atomique, DASE/LDG, Bruyères le Chatel, France
}

\begin{abstract}
TARANIS (Tool for the Analysis of Radiations from lightNings and Sprites) is a CNES satellite project dedicated to the study of the impulsive transfer of energy between the Earth's atmosphere and the space environment, and more precisely to the physics of Transient Luminous Events (TLEs) and Terrestrial Gamma-ray Flashes (TGFs). The scientific background and the scientific payload have been described by Blanc et al. [1] and Lefeuvre et al. [2], respectively. The present note is an extended summary of paper 2 , with a few updated information items.
\end{abstract}

Keywords: TLEs, TGFs, lightning induced electron precipitation (LEP), runaway electron beams, wave fields, fractional hop whistlers, effects on the atmosphere

PACS: $92.60 . \mathrm{hx}$

\section{INTRODUCTION}

TARANIS (Tool for the Analysis of Radiations from lightNings and Sprites) is a CNES satellite project dedicated to the study of impulsive transfers of energy between the Earth's atmosphere and the space environment, and more precisely to the physics of Transient Luminous Events (TLEs) and Terrestrial Gamma-ray Flashes (TGFs). Its main difference, compared with other space projects, is the simultaneous observation of wave fields and charged particles from a satellite which allows high sensitivity measurements. Presently, the project is completing its phase B study, with launch being expected in 2012 .

The scientific background and the scientific payload have been described by Blanc et al. [1] and Lefeuvre et al. [2], respectively. This extended abstract is an extended summary of paper 2 , with a few updated information items. It presents: (i) the science objectives, (ii) the instrumentation, and (iii) the contributions to major scientific issues and requirements for complementary measurements.

\section{SCIENCE OBJECTIVES}

The science objectives of the TARANIS mission fall into three broad categories:

(1) Advance physical understanding of the links between TLEs, TGFs and environmental conditions:

- location of the respective geographical positions and altitudes of TLE and TGF source regions,

CP1118, Coupling of Thunderstorms and Lightning Discharges to Near-Earth, edited by N. B. Crosby, T.-Y. Huang, and M. J. Rycroft (1) 2009 American Institute of Physics 978-0-7354-0657-5/09/\$25.00 
- identification of parent lightning flashes and associated electromagnetic emissions,

- investigation of possible correlations with: geomagnetic activity, large scale ionospheric perturbations, cosmic ray Extensive Atmospheric Showers (EAS), micrometeorites, etc.

(2) Provide other potential signatures of the impulsive transfer of energy to test generation mechanisms:

- bursts of Lightning induced Electron Precipitation (LEPs) and of Runaway electron Beams (RBs),

- associated electromagnetic or/and electrostatic fields.

(3) Provide inputs for modelling the effects on the Earth's atmosphere of TLEs, TGFs and bursts of precipitated and accelerated electrons:

- amount of energy released by TLEs, TGFs, LEPs, RBs in the atmosphere

- physical characteristics of the different ty pes of streamers.

\section{INSTRUMENTATION}

\section{The Instruments}

The TARANIS scientific payload is composed of six scientific instruments: MCP (Micro-Cameras and Photometers), XGRE (X-ray, Gamma-ray and Relativistic electron experiment), IDEE (Instrument Détecteur d'Electrons Energétiques), IME-BF (Instrument de Mesure du champ Electrique - Basse Fréquence), IME-HF (Instrument de Mesure du champ Electrique - Haute Fréquence), IMM (Instrument de Mesure du Champ Magnétique), plus a Multi Experiment Interface Controller (MEXIC).

$\boldsymbol{M C P}$ - The MCP instrument (E. Blanc, CEA/LDG, F + Univ.Tohoku-Hokkaido, JAXA J) makes optical observations in the nadir. It includes: (i) a lightning camera $(600-900 \mathrm{~nm})$ and a TLE camera $(762 \pm 5 \mathrm{~nm})$ with 30 images/s and $512 \times 512$ pixels per image, the observation zone and resolution at ground are, respectively, $\sim 500 \mathrm{~km}$ and $\sim 1 \mathrm{~km}$, (ii) three photometers $(762 \pm 5 \mathrm{~nm}, 337 \pm 5 \mathrm{~nm}, 150$ to $280 \mathrm{~nm})$ with an observation disk of $\sim 275 \mathrm{~km}$ radius, and a fourth photometer $(600$ to $900 \mathrm{~nm})$ with an observation disk of $700 \mathrm{~km}$ radius.

$\boldsymbol{X G R E}$ - the XGRE instrument (P.L. Blelly, CESR, F + APC, F) has as its objective the measurement of photon energies from $20 \mathrm{keV}$ to $10 \mathrm{MeV}$ and of relativistic electrons from 1 to $10 \mathrm{MeV}$. The instrument has been modified since the publication of paper 2, and a new design is under study.

IDEE - the IDEE instrument (J.A. Sauvaud, CESR/CNRS, F + Univ Prague, Cz) provides high resolution energetic spectra $(70 \mathrm{keV}-4 \mathrm{MeV})$ over a large dy namic range of fluxes and pitch-angle distribution. It consists of two spectrometers (each with a maximal field of view of $140^{\circ} \times 150^{\circ}$ ), one with a viewing axis making an angle 
of $60^{\circ}$ with the nadir, the second making an angle of $30^{\circ}$ with the anti-nadir direction. The angular resolution of each spectrometer is better than $35^{\circ}$.

$\boldsymbol{I M E} \boldsymbol{B} \boldsymbol{B} \boldsymbol{F}$ - the IME-BF instrument (E. Seran, LATMOS/CNRS, F + GSFC, USA) has the objective of identifying of upcoming $0+$ (fractional hop) whistlers associated with parent lightning flashes and monitoring the electromagnetic environment below 1 $\mathrm{MHz}$. It measures one component of the electric field (from $\mathrm{DC}$ to $1 \mathrm{MHz}$ ), and includes an ion probe.

$\boldsymbol{I} \boldsymbol{M} \boldsymbol{E}-\boldsymbol{H} \boldsymbol{F}$ - the IME-HF instrument (J.L. Rauch, LPCE/CNRS, F + Univ. Prague, IAP, $\mathrm{Cz}$ ) has the objective of detecting the HF/VHF electromagnetic signatures of lightning and other natural emissions. It measures one component of the electric field in the frequency band $100 \mathrm{kHz}-35 \mathrm{MHz}$. The sampling rate imposes severe onboard data selections.

IMM - the IMM instrument (J.L. Pinçon, LPCE/CNRS + Univ. Stanford, USA), has objectives in common with IME-BF. Below $20 \mathrm{kHz}$ it provides estimates of the propagation characteristics of electromagnetic waves. It includes a 3 axis VLF magnetic sensor $(0.001-20 \mathrm{kHz})$, a 1 axis magnetic sensor up to $1 \mathrm{MHz}$, and a $0+$ whistler detector.

MEXIC - the MEXIC instrument (M. Parrot, LPCE/CNRS + CBK, P) powers the scientific instruments and provides all interfaces between the scientific instruments and the satellite.

\section{The mission profile}

The mission profile is very similar to that of DEMETER. TARANIS is expected to be a two-year mission. It will fly in a $98^{\circ}$ polar orbit at altitudes $\sim 700 \mathrm{~km}$. It will be Sun-synchronous with a weak drift in local time. According to the present launcher opportunities, there is a high probability for operations around $22 \mathrm{LT}$.

The scientific payload is operated as a single instrument. It is systematically switched on from $-60^{\circ}$ to $+60^{\circ}$ geographical latitudes with two exceptions: (a) the optical instruments are switched off during the daytime, (b) the $\mathrm{X}$ and gamma rays instrument and the optical instruments (the cameras at least) are switched off over the South Atlantic Anomaly (SAA). Two operational modes have been defined:

- a low time resolution "survey" mode, in order to provide continuous monitoring

- a high time resolution "event" mode, in order to acquire and transmit data from all the instruments when a priority event is detected. The main triggering instruments are the photometers and the XGRE instrument. Triggering by other instruments is possible. 


\section{CONTRIBUTIONS TO MAJOR SCIENTIFIC ISSUES AND REQUIREMENTS FOR COMPLEMENTARY MEASUREMENTS}

Source regions of TLEs and TGFs - The TARANIS scientific payload will identify and characterize the source regions of TLEs, TGFs, and the 0+ whistlers associated with parent lightning flashes. Complementary ground-based and balloonbased measurements will be needed to check the TLEs and TGFs source regions, respectively, and measure complementary parameters. Measurements on the International Space Station will allow comparison observations made by TARANIS around 22LT with other observations at other LTs.

Characterization of EM signatures - Wave field measurements in an electromagnetic environment expected to be similar to that of DEMETER [3] will allow: a monitoring of natural and man-made emissions from DC to $35 \mathrm{MHz}$, discriminations between electrostatic and electromagnetic emissions from $0.1 \mathrm{~Hz}$ to $1 \mathrm{MHz}$, and estimations of the propagation characteristics up to $20 \mathrm{kHz}$. Complementary ground-based measurements are needed to compare lightning detection at the ground and in space, and to explain the ELF events detected at the ground. Balloon-borne experiments are expected to characterize the structure of the electrostatic fields above the thunderstorms.

Input parameters for generation models - Simultaneous observations and the characterization of TLEs, TGFs, 0+ whistlers, variations in ion density, Lightning induced Electron Precipitation, Runaway electron Beams, etc., on board TARANIS will study the full atmosphere / ionosphere / magnetosphere system. Simultaneous measurements on the ground, on balloon-borne experiments and on other spacecraft will be essential to: distinguish between correlations and coincidences, estimate variations in the $\mathrm{D}$ and $\mathrm{E}$ layers of the ionosphere, and estimate the amount of energy released within the atmosphere.

Modelling effects on the atmosphere - In the absence of measurements of atmospheric species on-board TARANIS, complementary atmospheric measurements are needed to evaluate the potential effects of the impulsive transfer of energy on variations of atmospheric species $\left(\mathrm{NO}_{\mathrm{x}}\right.$ and $\mathrm{O}_{3}$ ), and more generally on atmospheric models. Atmospheric species need to be measured on balloon-borne experiments or on other spacecraft missions.

* The TARANIS Team (PIs and lead CO Is) : P.L. Blelly, E. Blanc, J. Blecki, T. Farges, U.S. Inan, F. Lebrun, F. Lefeuvre, M. Parrot, R. Pfaff, J.L. Pinçon, Z. Nemecek, J.L. Rauch, O. Santolik, ,J.A. Sauvaud, E. Seran, M. Sato, M. Suzuki, Y. Takahashi. 


\section{REFERENCES}

1. E. Blanc, F. Lefeuvre, R. Roussel-Dupré, J.A. Sauvaud, Adv. Space Res., 2007, 40, 1268 -1275.

2. F. Lefeuvre, E. Blanc, J.L. Pinçon, R.Roussel-Dupré, D. Lawrence, J.A. Sauvaud, J.L. Rauch, H. de Feraudy, D. Lagoutte, Space Sci. Rev., 2008, $137: 301-315$.

3. J.J. Berthelier, M. Godefroy, F. Leblanc et al., Planet. Space Sci. (2006), 54, 456.

\section{ACKNOWLEDGMENTS}

The authors thank the referee for very useful suggestions and remarks. 